\title{
Verschuldung, Verschuldungskrisen und Anpassungsfähigkeit von Entwicklungsländern
}

\author{
von Joachim Betz
}

\section{Einleitung}

Die nicht erdölexportierenden Staaten der Dritten Welt sahen sich in den Jahren nach 1978 erneut einer Reihe von schweren außenwirtschaftlichen Belastungen gegenüber, die ihre wirtschaftlichen Aussichten verschlechterten.

a) Da ist zunächst einmal der zweite "Ölschock" (1979/80) zu nennen, der die Olpreise um ca. $115 \%$ und die Olimportrechnungen dieser Länder um 51 Mrd. \$(1978-81) steigerte. ${ }^{1}$

b) Im wesentlichen durch die Rezession in den Industrieländern verursacht (und durch die rezessionsbedingt zunehmenden protektionistischen Tendenzen dort), sanken das Exportvolumen, die terms of trade und damit die reale Kaufkraft der Exporterlöse dieser Länder erheblich; letztere von einem jährlichen Wachstum 1976-78 in Höhe von $10 \%$ auf 4-5 \% 1980-81.2 Am stärksten davon betroffen sind die auf die Märkte der Industrieländer ausgerichteten europäischen und asiatischen Entwicklungsländer und die überwiegend Komplementärprodukte (i. e. Rohstoffe) herstellenden Volkswirtschaften der Dritten Welt. Konnte man bis einschließlich 1978 noch eine gewisse Abkoppelung des Exportwachstums der NOPECs vom Wirtschaftswachstum in den Industrieländern beobachten - bedingt durch die Ausweitung des Süd-Süd-Handels und durch Steigerung der Marktanteile ${ }^{3}$ - so scheint die Rezession im "Norden" nun stärker auf den "Süden" durchzuschlagen.

c) Als Folge der sinkenden Kaufkraft ihrer Exporterlöse konnten die Importe 1980 und 1981 nur noch um jeweils $3 \%$ gesteigert werden und dies auch nur unter Hinnahme weiterer Leistungsbilanzverschlechterung. Die Abhängigkeit des Wirtschaftswachstums in Entwicklungsländern von Importen ist zwar geringer, als oft unterstellt, gleichwohl ist offenkundig, daß die Fähigkeit der NOPECs zur weiteren Importkompression (als eine mögliche Anpassungsstrategie) deutlich begrenzt sind.

Zahlenangaben in: IMF, World Economic Outlook, Washington, D.C., June 1981, Tabelle S. 122.

Ebenda, S. 119

Vgl. OECD, Development Co-operation, 1980 Review, Paris, 1980, S. 77 
d) Die 70er Jahre sind durch die bekannte und oft beschriebene Privatisierung der Definzitfinanzierung der Entwicklungsländer ${ }^{4}$ gekennzeichnet, die insbesondere den Anteil der von den internationalen Banken gehaltenen Forderungen erhöht hat. Damit war zwangsläufig eine Verkürzung der durchschnittlichen Kreditlaufzeiten und eine deutliche Erhöhung der Zinskosten verbunden (s. u.), die sich noch durch den in den letzten Jahren verfolgten Antiinflationskurs in den Industrieländern verschärft hat. Seit 1979 ist allerdings der Anteil der Finanzinstitutionen an der Schuldenaufnahme der NOPECs zugunsten von Entwicklungshilfemitteln, aber auch zugunsten von Reservenauflösungen, IMF-Ziehungen und kurzfristigen Krediten wieder leicht rückläufig. ${ }^{5}$

e) Nichtsdestoweniger müssen die Entwicklungsländer einen bedeutend gestiegenen Schuldendienst bezahlen. Dieser steigerte sich von 1978-81 von 43 auf 96 Mrd. \$ und entspricht damit volumenmäßig über $80 \%$ der Leistungsbilanzverschlechterung in diesem Zeitraum. Entsprechend erhöhte sich die Schuldendienstquote von 13,8 \% (1977) auf 20,8\% (1981). ${ }^{6}$ Genausowenig wie beim Olschock, läßt sich aus der zahlenmäßigen Gegenüberstellung Kapitallasten/Zahlungsbilanzdefizit der Schuldige für die wachsenden außenwirtschaftlichen Probleme der NOPECs umstandlos dingfest machen. ${ }^{7}$ Man darf nämlich nicht vergessen, daß bei der üblichen Überrollung der Bankkredite an echten Zusatzbelastungen für die Entwicklungsländer nur die (auch nicht ganz unbeachtlichen) Zinskosten zu Buche schlagen.

Alle bisherigen Einschränkungen beiseite, müssen schwächer steigende Exporte, sich verschlechternde terms of trade, ein geringes, aber nach wie vor steigendes Importvolumen und höhere Kapitallasten sich zwangsläufig in einer Verschlechterung der Leistungsbilanz niederschlagen. Die entsprechenden Defizite wuchsen denn auch von 38 Mrd. \$(1978) auf 82 Mrd. \$ (1980) und werden für 1982 auf $102 \mathrm{Mrd}$. \$ geschätzt. ${ }^{8}$ Es ist nun aber eine Binsenweisheit, daß sich hohe Defizite nur durchhalten lassen bzw. überhaupt erst möglich sind, wenn sie finanziert werden. Die steigende Passivierung der Leistungsbilanzen der Länder der Dritten Welt in den letzten zehn Jahren hatten also das Wachstum und die Diversifikation der Angebotsquellen finanzieller Ressourcen zur notwendigen Voraussetzung. Ebenso ist die steigende Verschuldung der Entwicklungsländer - die gerne mit Negativbegriffen wie "Last ", "Berg" o. ä. assoziiert wird, notwendige Folge der nicht durch eigene Exporte und Entwicklungszuschüsse gedeckten Importe, die zur Erzielung einer geplanten Wachstumsrate nötig sind. Sofern die Rendite der

Statt vieler: Siegfried Schultz, Zur Verschuldungslage der Dritten Welt, in: DIW Vierteljahresheft 1/1981; Manfred Holthus, Verschuldung und Verschuldungsfähigkeit von Entwicklungsländern, in: Hamburger Jahrbuch für Wirtschafts- und Gesellschaftspolitik, 26. Jahr (1981), S. 239-255; Veronika Büttner, Externe Verschuldung von Entwicklungsländern: Konfliktbereiche und Lösungsmöglichkeiten im Rahmen einer NordSüd-Politik, SWP - S. 294, Februar 1982.

Angaben in Anm. 1, S. 129 und in OECD, Development Co-operation, 1981 Review, Paris 1981, S. 60.

Angaben in Anm. 1, S. 135.

Wie dies in der neuerlichen Rede vom "Zinsschock" of t geschieht.

Anm. 1, S. $43 \mathrm{f}$. 
eingesetzten Fremdmittel in den Entwicklungsländern zumindest die Zinskosten (in Auslandswährung) erbringen, ist dies ein durchaus wünschenswerter Vorgang.

\section{Möglichkeiten des Zahlungsbilanzausgleichs}

Eng damit zusammenhängend kann man eine übermäßige Herausstellung der Schuldensituation bei der Behandlung der gegenwärtigen außenwirtschaftlichen Probleme der Dritten Welt konstatieren. Nun ist aber der Anstieg der Verschuldungsquote nur ein Reflex einer über die vorhandenen Ressourcen hinausgehenden Nachfrage und der wirtschaftlichen/politischen Restriktionen alternativer Möglichkeiten des Leistungsbilanzausgleichs durch

a) Erhöhung der Exporte bzw. Inlandsproduktion bisheriger Importe;

b) Maßnahmen zur Verbesserung der Kapitalproduktivität;

c) stärkere Eigenfinanzierung der Investitionen durch Erhöhung der internen Sparrate;

d) Verringerung der internen Absorption (i. e. Nachfragedämpfung) durch monetäre und/oder fiskalische Restriktionspolitik.

Erst wenn diese Möglichkeiten ausscheiden, sei es, weil sie bereits ausgeschöpft sind, sei es, weil sie intern nicht konsensfähig sind, steigt notwendigerweise die Verschuldung. Umgekehrt kann man in gleicher Weise nicht von außenwirtschaftlichem Gleichgewicht sprechen, wenn zwar die Verschuldung stagniert, der Ausgleich der Zahlungsbilanz aber nur durch übermäßige und politisch möglicherweise krisenhafte Wachstumsdrosselung zuwege gebracht wird.

Aussagen über die Fähigkeit der Entwicklungsländer zur realen Anpassung an die "externen Schocks" können auf aggregierter Ebene kaum gemacht werden. Allenfalls kann man sagen, daß ihre geringeren Exportmöglichkeiten, sowie die gestiegenen Energieund Zinskosten einen enormen Anpassungsbedarf hervorrufen, sollen Wirtschaftswachstum und Kreditwürdigkeit aufrecht erhalten werden. Es fragt sich, ob die zur realen Anpassung nötigen Schritte - die im wesentlichen Konsumverzicht bedeuten - politisch überhaupt durchgesetzt werden können. Scheitern aber die Regierungen der Entwicklungsländer an dieser Aufgabe, steigen bei der gegenwärtigen Struktur der Finanzierungsströme sowie den mittelfristig wohl eher mäßigen globalen Konjunkturaussichten die Risiken von Verschuldungskrisen in der Dritten Welt.

\section{Allgemeine Kapitalangebots- und Bedienungsprobleme?}

Aus dem Vorgenannten sowie v. a. natürlich aus dem Wachstum, der veränderten Struktur und der schlechteren Bedingungen der aufgenommenen Schulden der Entwicklungsländer erklären sich die heftigen Befürchtungen, mehr und mehr Entwicklungsländer wären künftig gar nicht mehr in der Lage, ihre Schulden zu bedienen, bzw. würden aufgrund der Aussichtslosigkeit ihrer Lage bewußt ihre eigene Zahlungsunfähigkeit herbei- 
führen. ${ }^{9}$ Der Bankrott eines oder weniger Großschuldner würde genügen, um den gesamten Recyclingprozeß der Banken nachhaltig zu stören mit der Folge nicht abzusehender finanzieller Kettenreaktionen. Verstärkt wurden diese Befürchtungen durch vermutete Engpässe auf der Kapitalangebotsseite. Zu letzterem zuerst. Der Anteil des Auslandsgeschäfts ist bei den international tätigen Banken deutlich gestiegen; mit der raschen Expansion der Kreditvergabe (1974 bis einschließlich 1979 sowie 1981) hielt die Eigenkapitalausstattung nicht Schritt. Das zukünftige Kreditpotential wird aber wesentlich - v. a. bei verschärfter Bankenaufsicht - durch dieses bestimmt. Schwierigkeiten für eine Verbreiterung der Kapitaldecke ergaben sich aus den (wegen der übermäßigen Liquidität der Euromärkte) zurückgehenden Gewinnen im Auslandsgeschäft, die die Kurse der Bankaktien nach unten drückte. ${ }^{10}$

Als hauptsächlicher begrenzender Faktor einer weiteren Ausdehnung des Kreditgeschäfts im Tempo der vergangenen Jahre wird allgemein die erhebliche Konzentration des Kreditportefeuilles der Banken angesehen: so lauteten Ende 1980 immerhin 70 \% der internationalen Bankforderungen auf nur zehn Schuldner und seit 1978 haben gar nur vier Länder $80 \%$ der Nettoneukredite erhalten. ${ }^{11}$ Die geringe Risikostreuung und das heftige unkontrollierte Wachstum der Eurokapitalmärkte haben in vielen Industrieländern (vor allem in den USA, aber auch in der Bundesrepublik, der Schweiz und Japan) Forderungen nach einer stärkeren bankenaufsichtlichen Kontrolle Nachdruck verliehen. Sie laufen darauf hinaus, die Transparenz des Kreditgeschäftes zu erhöhen und den Anteil der nationalen Banken am internationalen Kreditgeschäft insgesamt und insbesondere gegenüber einzelnen Schuldnern zu begrenzen. ${ }^{12}$

Daneben bestehen Befürchtungen, das Kapitalangebot für die Entwicklungsländer könne auch durch konkurrierende Nachfrage der Industrieländer vermindert werden, sei doch mit einem langsameren Abbau der Leistungsbilanzdefizite dieser Länder als nach dem ersten Olschock und gleichzeitig mit hohem Bedarf an energiesparenden Investitionen zu rechnen.

Dauerhaft haben sich die (übrigens periodisch wiederkehrenden ${ }^{13}$ ) Befürchtungen sinkender Kapitalangebotsfähigkeit der Banken nicht bewahrheitet; das Tief an Neuzusagen an die NOPECs 1980 wurde von einem Rekordzusagevolumen 1981, dieses wiederum von einem kräftigen Einbruch in der ersten Jahreshälfte $1982^{14}$ abgelöst. Die Eigen-

9 In diesem Sinne: Charles Lipson, The International Organization of Third World Debt, in: International Organization, vol. 35, no. 4 (Autumn 1981), S. 603-631; Surinder K. Malik, Bleak Prospects for Commercial Bank Lending to Developing Countries, in: Außenwirtschaft, Jg. 35, Heft III (Sept. 1980), S. 249-266; Chandra Hardy, Rescheduling Developing Country Debts, in: The Banker, July 1981, S. 33-38.

10 Zahlen hierzu bei Arturo C. Porzecanski, The International Financial Role of U.S. Commercial Banks: Past and Future, in: Journal of Banking and Finance vol. 5, no. 1 (1981).

11 Angaben in: D. Gorman, A New Role for the IMF? in: Barclays Review, S. $33 \mathrm{f}$.

12 Dazu: Surinder K. Malik, Anm. 9; Geschäftsbericht der Deutschen Bundesbank für das Jahr 1979, S. 52 ff.

13 Siehe IMF, Occasional Paper no. 1, International Capital Marktes - Recent Developments and Short-Term Prospects, Washington, D.C., Sept. 1980, S. 7.

$14 \mathrm{Vgl}$. Geschäftsbericht der Deutschen Bundesbank für das Jahr 1981; World Financial Markets, Dec. 1981, S. 8 ff.; NZZ v. 14. 1. 1982, Beginn eines neuen Kapitels a m Eurokreditmarkt; FAZv. 20. 1. 1982, Uber 200 Milliarden Dollar neue Eurokredite; Euromoney, May 1982, The Shunning of the Sovereign Borrower. 
kapitaldecke der Banken ist nur geringfügig dünner geworden, zudem macht das Auslandsgeschäft der Banken weniger als ein Fünftel ihres Gesamtumsatzes aus (wovon Kredite an die NOPECs wiederum nur einen Anteil von ca. $30 \%$ haben) und das Ausfallrisiko war im internationalen Geschäft bisher geringer als im Inlandsgeschäft. ${ }^{15}$ Die konkurrierende Kreditnachfrage der Industrieländer hielt sich bisher (rezessionsbedingt) im Rahmen und die rituellen Beschwerden der Banken über zu geringe Profitmargen gehören zum Geschäft. Wären sie völlig berechtigt, müßte man sich fragen warum die Banken dennoch an Entwicklungsländer leihen, wozu sie ja nicht gezwungen sind. Größere Zurückhaltung der Banken würde bei gleichbleibender Kreditnachfrage alsbald die "Zinsmargen" wieder in die Höhe treiben und damit risikokompensierend wirken. ${ }^{16}$

Die übermäßige Konzentration des Kreditportefeuilles auf Einzelschuldner stellt sicher ein Problem dar, aber nicht vornehmlich deswegen, weil die Hauptkreditnehmer besonders insolvenzanfällig wären (s. u.). Vielmehr rührt die Gefahr daher, daß die Schuldnerländer ihre fälligen Kredite durch Neuaufnahmen "bezahlen ", - auf deren gleichbleibenden Fluß sie angewiesen sind; in dem Maße aber wie Kredite nur noch überrollt werden, sind den Banken die Hände für neue Engagements gebunden. Bei dem geringen Anteil der Eurokredite an die NOPECs an den Aktiva der Banken genügt eine nur geringe Änderung ihrer Risikoperzeption um auch von der Angebotsseite her die weniger kreditwürdigen Entwicklungsländer in erhebliche Schwierigkeiten zu bringen. ${ }^{17}$

Befürchtungen, die Entwicklungsländer seien generell total überschuldet und könnten den Kapitaldienst - vor allem unter den gegenwärtigen widrigen weltwirtschaftlichen Umständen - nicht oder nur unter untragbaren innenpolitischen Friktionen erbringen und würden daher eine Art kollektiver Zahlungsverweigerung vorziehen, sind $\mathrm{m}$. E. wenig berechtigt. Der schnelle Schuldenwachstum der 70er Jahre war nämlich zu einem guten Teil inflationsbedingt. Real fiel es geringer aus als im Jahrzehnt vorher. Bezogen auf die üblichen volkswirtschaftlichen Größen (Währungsreserven, BIP, Außenhandel) blieb die Verschuldung der NOPECs nahezu konstant. Úberdies kann man ab 1979 eine deutliche Verlangsamung des Schuldenwachstums konstatieren, freilich bei Auflösung der Währungsreserven und erheblicher Zunahme der kurzfristigen Kredite. Signifikant erhöht (auf ca. 20,8 \%) hat sich aber der Schuldendienstquotient, also das Verhältnis von Schuldendienst zu Gütern und Dienstleistungen. ${ }^{18}$ Das rapide Wachstum des Schulden-

15 Siehe dazu A. C. Porzecanski, Anm. 10; IMF, Occasional Paper no. 1, Anm. 13 S. 9; World Bank, World Development Report 1981, Washington, D.C., 1981, S. 61.

16 Dazu: Alexander Fleming, Private Flows to Developing Countries and their Determination: Historical Perspectives, Recent Experience, and Future Prospects, World Bank Staff Working Paper no. 484, August 1981, S. 33 .

17 Group of Thirty, Balance-of-Payments Problems of Developing Countries, New York, 1981, S. 8 f., IMF, Occasional Paper no. 3, External Indebtedness of Developing Countries, Washington, D.C., May 1981, S. 10.

18 Zur Problematik: Millard Long und Frank Veneroso, The Debt Related Problems of the Non-Oil Less Developed Countries, in: Economic Development and Cultural Change, vol. 29, o. 3 (April 1981); Zahlenangaben: IMF, Occasional Paper no. 3, Anm. 17, S. 5; Anm. 1, Tabelle 30; Nicholas C. Hope, Developments in and Prospects for the External Dbt of the Developing Countries: 1970-1980 and Beyond, World Bank, Staff Working Paper no. 488, August 1981. 
dienstes (1971-81 $26 \%$ p. a.) ist natürlich ein Ausfluß der weiter oben geschilderten verschobenen Kreditquellenstruktur zugunsten der Finanzinstitutionen und den in den letzten Jahren steigenden Euromarktzinsen. Es hat sich aber 1981 bereits wieder leicht verlangsamt (auf $22 \%)^{19}$ und ist zumindest zum Teil durch den Wertverfall des Dollars und vorzeitige Rückzahlungen (angesichts der günstigen Refinanzierungskonditionen 1977-78) überzeichnet. Man darf auch nicht vergessen, daß der Schuldendienst zu $60 \%$ aus Tilgungszahlungen besteht, die, Kreditwürdigkeit vorausgesetzt, in der Regel durch neue Zusagen überrollt werden. Die realen Kosten des Schuldendienstes sind zudem bislang - gemessen an den globalen Inflationsraten - für die meisten Entwicklungsländer negativ gewesen. Das rührt daher, daß die offiziellen Transfers und auch die Lieferantenkredite deutlich unter den Inflationsraten liegende Zinssätze aufwiesen und auch die vor 1974 zugesagten Euromarktkredite die nachfolgende Inflationsentwicklung nur unvollständig antzipierten. So stiegen die durchschnittlichen Zinskosten auf alle Kapitaltransfers für die ärmeren Entwicklungsländer (LICs) 1971 bis 1981 von 2,8 auf 8,6\% p. a. und nur bei den Schwellenländern von 7,0 auf 13,3\% und damit deutlich in die Zone real positiver Raten. ${ }^{20}$

Dazu kommt, daß viele Entwicklungsländer ihre Währungsreserven selbst an den Eurogeldmärkten plaziert haben. Rechnet man nun nur ihre Ausleihungen zu variablen Zinssatz und ihre Einlagen am selben Markt gegeneinander auf, so verbleiben nur wenige Länder, die substantielle Nettoschuldner hochverzinslicher Eurokredite sind (vor allem Brasilien, Südkorea, Chile, die Elfenbeinküste, Marokko, Mexiko und die Philippinen). ${ }^{21}$ Zuletzt handelt es sich bei den gerne herausgestellten Ländern mit den größten Rückzahlungslasten ${ }^{22}$ entweder um ölexportierende Entwicklungsländer oder um solche, die dauernd oder wenigstens zeitweise (vor allem auch nach dem zweiten Olschock) eine erhebliche wirtschaftliche Anpassungsfähigkeit bewiesen haben (Prototyp: Brasilien). Trotzdem wäre es unsinnig, wegen der geringen Wahrscheinlichkeit einer die gesamte Dritte Welt betreffenden Schuldenkrise Verschuldungsoptimismus hinsichtlich aller einzelnen Länder abzuleiten. Einmal verdeckt die Aggregatdatenbetrachtung nämlich die stark uneinheitliche Entwicklung: gemessen an der Schuldendienstquote hat sich die Lage vieler traditionell kreditsuchender Länder (etwa Indien, Pakistan, Ägypten, Indonesien etc.) von 1975 bis 1981 gebessert oder gehalten, während bei anderen ein deutlicher Anstieg der Quote zu verzeichnen ist. Diese gegenläufigen Tendenzen führen dazu, daß die Aggregatzahlen keine krisenhafte Verschlechterung signalisieren, während doch die Lage für eine ganze Reihe von Ländern zunehmend prekärer wird. Symptome hierfür sind die in den letzten fünf Jahren deutlich steigenden Zahlungsrückstände und die sich mehreren Umschuldungen im Rahmen des Pariser Klub und durch die Banken. ${ }^{23}$

19 Anm. 1, Tabelle S. 135.

20 Vgl. dazu: OECD, External Debt of Developing Countries, Paris, 1981, S. 7.

21 Vgl. World Bank, World Development Report 1981, S. 52.

22 Brasilien, Mexiko, Venezuela, Algerien, Spanien, Saudi Arabien, Südkorea, Jugoslawien, Argentinien, Indonesien (in dieser Reihenfolge).

23 Angaben hierzu im Economist v. 20. 3. 1982, International Banking - Survey; Chandra Hardy, Anm. 9. 
Die Defizitfinanzierung 1979/80 geschah überdies in starkem Maße durch Reservenabbau, weitere Importkompression und die Aufnahme kurzfristiger Kredite: ein nicht beliebig fortsetzbares Verfahren. Der Verweis auf die durchschnittlich bisher real negativen Zinsen für den Großteil der Klienten kann auch umgekehrt werden: Ist es den Entwicklungsländern schon unter diesen Finanzierungsbedingungen nicht gelungen, ihre Verschuldungssituation zu verbessern, was eine für bescheidene Produktivität der eingeworbenen Mittel bzw. ihre hauptsächlich konsumptive Verwendung spricht, so ist bei den heute deutlich höheren Refinanzierungskosten noch weniger damit zu rechnen. Müssen insgesamt also behauptete allgemeine Kapitalangebots- und -bedienungsprobleme zurückgewiesen werden, so kommt es bei der Labilität des privaten Ressourcentransfers darauf an, daß die Länder, die als besonders gefährdet gelten müssen, ihre wirtschaftliche Performanz verbessern und sich an die verschlechterten externen Bedingungen anpassen. Ein zu ausschließlicheres Vertrauen auf die kreditfinanzierte "Anpassung" wie in den Jahren 1974 ff. hätte sicherlich fatale Folgen.

\section{Wert und Unwert von Verschuldungsindikatoren}

Aus dem Schuldenwachstum der Entwicklungsländer (und natürlich auch der Staatshandelsländer) und der beobachteten Unterschiede im Grad der Verschuldung und der Bedienungsprobleme erklärten sich die periodisch immer wieder heftiger werdende Suche nach Indikatoren und Indikatorsystemen, die es erlauben zwischen kritischen Fällen, meist gleichgesetzt mit umgeschuldeten Ländern, und unkritischen zu unterscheiden. Uber die Sinnhaftigkeit von Einzelindikatoren oder Indikatorsystemen zur Abschätzung der Kreditwürdigkeit soll hier nicht ausführlich diskutiert werden. ${ }^{24}$ Es sei nur soviel ausgesagt, daß die prognostische Fähigkeit dieser Systeme (und noch viel mehr der Einzelindikatoren) sich als relativ gering erwiesen hat und sie insbesondere für die laufende Periode bereits wesentlich mehr Verschuldungskrisen anzeigen, als tatsächlich eintraten. ${ }^{25}$ Die Schwächen hängen einmal mit Datenproblemen zusammen (zu späte und unvollständige Erfassung), vor allem aber mit der Tatsache, daß die Indikatoren auf eine sehr kurzfristige Betrachtung abstellen und deshalb für die Laufzeiten der meisten Kredite uninteressant sind. Denn inwieweit ein über externe Mittel verfolgter Industrialisierungsweg durchgehalten werden kann ist doch weniger von statischen Größen, sondern von der wirtschaftspolitisch beeinflußbaren Entwicklungsdynamik der internen Sparquote, der Exporte und der Produktivität des eingesetzten Kapitals abhängig sowie von den weltwirtschaftlichen Rahmenbedingungen (Refinanzierungsmöglichkeiten, Wachstum des Welthandels) während des Rückzahlungszeitraums. ${ }^{26}$ Damit ist letztlich auch

24 Statt vieler: Rainer Erbe und Susanne Schattner, Indicator Systems for the Assessment of the External Debt Situation of Developing Countries, in: Intereconomics, Nov./Dec. 1980, S. 285 ff.

25 Ebenda.

26 Dazu auch IMF, Occasional Paper no. 3, Anm. 17, Appendix III. 
die Frage nach den gerne als Randbedingung behandelten internen politischen Verhältnissen und ihrer Veränderung auf die Verschuldungsentwicklung gestellt. Es ist aber völlig offenkundig, daß nicht voraussehbare politische "Störungen" in einigen Ländern entscheidender für das Eintreten krisenhafter außenwirtschaftlicher Entwicklungen waren, als das Volumen, die Struktur und die Bedingungen der vor diesem Zeitpunkt aufgenommenen Auslandskredite.

\section{Allgemeine Charakteristika verschuldungsgefährdeter Länder}

Die statt dessen oft geforderten detaillierten wirtschaftlich-politischen Länderanalysen und -prognosen findet man aber freilich recht selten, zumal dann, wenn man von den Länderberichten des IMF und der Weltbank absieht. Unbefriedigend wäre das alleinige Abstellen auf die Besonderheiten der Einzelfälle aber auch deswegen, weil daraus leicht der Schluß gezogen werden könnte, über die Anpassungsfähigkeit der EL an widrige außenwirtschaftliche Umstände ließen sich überhaupt keine verallgemeinerungsfähigen Aussagen machen. Darauf zielen die nun folgenden Ausführungen. Es wird gefragt werden

a) ob sich Länder/Ländergruppen identifizieren lassen, die eine geringe Anpassungskapazität an verschlechterte außenwirtschaftliche Bedingungen aufweisen und die daher - sofern sie Kredite erhalten - notwendigerweise in Verschuldungsschwierigkeiten geraten;

b) welche spezifischen Eigenheiten der Wirtschaftsstruktur, des internen Wirtschaftsmanagements und der dahinterliegenden politischen und gesellschaftlichen Verfassung der Länder dafür maßgebend sind, daß nicht-schuldentreibende Anpassungsschritte nicht oder nicht rechtzeitig unternommen wurden oder unternommen werden konnten;

c) wie von diesem Befund her das internationale Instrumentarium zur Vermeidung und zur Bearbeitung von Schuldenkrisen in Entwicklungsländern zu beurteilen ist.

Auf einer ersten und vordergründigen Ebene kann man nun versuchen, die Charakteristika der Länder und ihrer eingeschlagenen Entwicklungswege zu untersuchen, die bisher umgeschuldet wurden. Es handelt sich hier um Länder, die

a) ein geringes Wirtschaftswachstum aufweisen;

b) über eine geringe interne Sparrate verfügen, etwa weil die Steuerstruktur rigide ist, ineffiziente Staatsbetriebe Ressourcen verschlingen, öffentliche Leistungen nicht kostendeckend abgegeben werden und die Sparzinsen weit hinter der Inflationsrate herhinken;

c) eine geringe Kapitalproduktivität aufweisen, wofür meist hoher Sozialkonsum, die geringe Rentabilität des öffentlichen Investitionsprogramms bzw. dessen lange Ausreifungszeit und ein allgemein hoher Staatsanteil am BSP maßgebend sind; 
d) ein schwaches Exportwachstum oder stark fluktuierende Exporterlöse haben, bei gleichzeitig stark ansteigenden Importen, wofür Importsubstitution bis in den Zwischengüterbereich, überbewertete Währungen und die Diskriminierung der Landwirtschaft verantwortlich sind;

e) ein mäßig effizientes Schuldenmanagement aufweisen, da zuviele Schulden zu ungünstigen Bedingungen und prozyklisch zur Verschärfung der Leistungsbilanzsituation aufgenommen wurden. (Dieser letzte Punkt zeigt bereits die Schwierigkeit auf, interne von externen Gründen bei Verschuldungskrisen zu trennen). ${ }^{27}$

Diese gemeinsamen Merkmale enthalten nicht viele Ubbrraschungen, da sie exakt jenen theoretischen Bedingungen widersprechen, deren Einhaltung "für die Erhaltung der langfristigen Rückzahlungsfähigkeit in einem growth-cum-debt Prozeß" gegeben sein muß, nämlich schnelles Wachstum, hohe marginale Sparquoten, über dem internationalen Zinssatz liegenden Erträge der investierenden Auslandsmittel und eine Zuwachsrate der Exporte, die nach Abzug des Schuldendienstes eine für die angezielte Wachstumsrate genügend hohe Importkapazität beläßt. ${ }^{28}$

Es wäre nun interessant zu sehen, ob sich mit der Verletzung der augenfälligen Stabilitätsbedingungen strukturelle wirtschaftliche Merkmale oder bestimmte Entwicklungsstrategien der in Zahlungsbilanzkrisen geratenden Länder korrespondieren, wie dies weiter oben gefragt wurde. Dabei sollen über den offensichtlichen Kreis der Umschuldungsfälle hinaus nach den Gemeinsamkeiten jener Länder gesucht werden, die eine geringe Anpassungsfähigkeit an die mit der ersten Olkrise einhergehenden externen Schocks aufwiesen, wobei Anpassungsfähigkeit im Sinne einer nicht die relative Verschuldung steigernden Erhaltung oder gar Verbesserung der realen Ressourcenbilanz ${ }^{29} \mathrm{zu}$ verstehen ist.

Die naheliegende Antwort nach den Ländern mit den größten Anpassungsproblemen an die externen Schocks wäre, daß dies wohl jene Staaten sein müßten, die auch die relativ schwersten außenwirtschaftlichen Lasten in dieser Zeit zu tragen hatten, ist auf der Grundlage der Menge und der Qualität der empirischen Evidenz falsch.

Eine ganze Reihe von Studien, die unter der Leitung von Bela Balassa angefertigt wurden, zeigen keinen positiven, sondern einen negativen Zusammenhang zwischen dem relativen (negativen) Zahlungsbilanzeffekt der externen Schocks (terms of trade-Verluste; geringer wachsendes Exportvolumen) und dem Wirtschaftswachstum und einen ebenfalls negativen Zusammenhang zwischen dem Rückgriff auf externe Finanzmittel zur

27 Dazu etwa: IMF, Occasional Paper no. 3, Anm. 17; Hans-Bernd Schäfer und Siegf ried Schultz, Der Zusammenbruch internationaler Zahlungsfähigkeit - Ursachen und Wirkungen, in: Hans-Bernd Schäfer (Hrsg.), Gefährdete Weltfinanzen, Bonn-Bad-Godesberg, 1980; Miguel S. Wionczek, LDC External Debt and the World Economy, Mexiko, 1978, Pierre Dhonte, La dette des pays en Développement, Paris, 1979.

$28 \mathrm{Vgl}$. Manfred Holthus, Anm. 4, S. 249.

29 Zur Definition und Modellbildung: Bela Balassa, The Newly-Industrializing Developing Countries af ter the Oil Crisis, World Bank Staff Working Paper no. 437, Washington D.C., Oct. 1980; ders., André Barsony und Anne Richards, The Balance of Payments Effects of External Shocks and of Policy Responses to these Shocks, OECD, Paris, 1981; Frederick Z. Jaspersen, Adjustment Experience and Growth Prospects of the Semi-Industrial Economies, World Bank Staff Working Paper no. 477, Washington D.C., August 1981. 
Bewältigung der externen Schocks und dem Wirtschaftswachstum. Entscheidend für den Einfluß der externen Schocks auf die Wirtschaftsentwicklung der untersuchten Länder waren nicht die Größe der Schocks, sondern ihre interne wirtschaftspolitische Verarbeitung. Vielfach wurden außenwirtschaftliche Störungen erst durch zusätzliche "interne Schocks" (= unangemessene Wirtschaftspolitik) zu einem gravierenden Problem (z. B. in Jamaika, Peru, Mexiko und Portugal), während andererseits negative externe Entwicklungen vielfach erst den Anstoß für eine eher durchhaltbare Wirtschaftspolitik gaben (z. B. in Chile, Uruguay, Portugal nach dem ersten Olschock und in Indien, Brasilien und der Türkei nach dem Zweiten), wobei über die politische Wünschbarkeit des neuen Kurses nichts ausgesagt werden soll. ${ }^{30}$

Andere Studien zeigen, daß in der Periode 1974-77 der weitaus wichtigste Faktor der sich verschlechternden Ressourcenbilanz eine über das vorhandene Angebot hinausgehender Nachfragestimulierung (meist durch öffentliche Investitionsprogramme) war, ${ }^{31}$ und daß sich ganz allgemein die Inflationsraten der NOPECs (vor allem in Afrika) bei sinkendem Wachstum erhöhten. ${ }^{32}$ In zwei neueren Arbeiten wird auch das gern gehörte Argument relativiert, die Olkrise sei vor allem am Wachstumseinbruch der Entwicklungsländer schuld. In geringem Maße trifft dies nur für die stärker industrialisierten und überwiegend weltmarktorientierten Staaten zu, die die Krise durch vermehrte Exporte aber alsbald absorbieren konnten. ${ }^{33}$

Positiv gewendet zeichnen sich die Länder mit hoher Anpassungsfähigkeit an die verschlechterten Rahmenbedingungen nach 1974 als jene aus, die weltmarktorientierte Strategie entweder weiter verfolgt haben oder zu diesen umgeschwenkt sind. Sie konnten sowohl ihre Marktanteile erhöhen als auch ihre Wachstumsraten einigermaßen halten und blieben im Ausmaß ihrer relativen Verschuldung in etwa auf dem vorherigen Stand. Gleichzeitig wiesen sie höhere Sparquoten und eine größere Kapitalproduktivität aus. Die Vermutung geht dahin, auch dies als Folge der Weltmarktorientierung (Technologieimport, Skalenerträge) und der notwendigerweise mit ihr verbundenen Wirtschaftspolitik (realistische Währungsparitäten und Zinssätze) zu sehen. ${ }^{34}$

Negativ gewendet stellen sich die Länder mit Binnenmarktorientierung und in den Zwischen- und Investitionsgüterbereich hinein getriebenen Importsubstitution als die größten Problemfälle heraus. Importsubstitution und Wirtschaftswachstum sind negativ miteinander korreliert; die Enge des inneren Marktes läßt eine derart verfolgte Industriali-

30 Siehe dazu die in Anm. 29 angegebene Werke von Balassa sowie ders., The Policy Experience of Twelve Less Developed Countries, 1973-1978, World Bank Staff Working Paper no. 449, Washington D.C., August 1981.

31 Siehe Stanley W. Black, The Impact of Changes in the World Economy on the Stabilization Policies in the 1970s, in: William R. Cline und Sidney Weintraub (Hrsg.), Economics Stabilization in Developing Countries, Washington, 1981; als Uberblick: Tony Killick, Extent, Causes and Consequences of Disequilibria in Developing Countries, ODl Working Paper no. 1, London, March 1981.

32 Vgl. IMF Survey, May 4, 1981.

33 Peter Nunnenkamp, The Impact of Rising Oil Prices on Economic Growth in Developing Countries in the Seventies, Kieler Inst. für Weltwirtschaft, unv. Ms., 1982; ders., Rising Oil Prices and Industrial Production in Some Developing Countries in the Seventies, Kieler Inst. für Weltwirtschaft, unv. Ms., 1982.

34 Vgl. Anm. 30. 
sierung zunehmend teurer und unproduktiver werden und erlaubt Importkompression bald nur noch unter Inkaufnahme von Produktionsverlusten und geringer Kapazitätsauslastung. Uberbewertete Wechselkurse und real negative Zinsen (zur Investitionssubventionierung) gehen mit der Importsubstitution meist einher. Exportschwäche, Kapitalintensivierung, Diskriminierung der Landwirtschaft und Begünstigung stärkerer staatlicher Wirtschaftskontrollen sind die logische Folge. ${ }^{35}$

Ein nicht unerheblicher Teil der Länder mit Anpassungsproblemen zeichnet sich auch durch vor der Krise unternommene, ambitionierte öffentliche Investitionsprogramme aus, die bei zunehmender Finanzklemme nur schwer zurückgefahren werden konnten. Eine die Importpreissteigerungen akkomodierende und zuweilen deutlich überkompensierende Geldpolitik (1979/80 bei immerhin 40 Entwicklungsländern der Fall) ${ }^{36}$ war für eine deutliche Inflationsbeschleunigung in den OPECs in den Jahren 1974/75 und 1979/ 80 verantwortlich. Es ist auch nicht uninteressant, daß die Höhe der beobachtbaren Inflationsraten negativ mit der wirtschaftlichen Offenheit korrelieren, externe Ursachen also kaum dafür verantwortlich gemacht werden können. ${ }^{37}$ Dieser Zusammenhang ist auch kaum verwunderlich, da permanent über die Paritätsänderungen hinausschießende Inflationsraten sich zwangsläufig (wettbewerbsbedingt) in stärkerer Binnenmarktorientierung niederschlagen müssen.

Interessant ist nun nicht die Feststellung von Binnenmarktorientierung und Geldmengenvermehrung an sich, sondern die Frage, warum Regierungen in Entwicklungsländern (offenbar wider besseres Wissen) diesen Entwicklungen ihren Lauf lassen. Dies rührt an die eigentlich interessierenden Probleme der internen Macht- und Einflußverteilung, die ein bestimmtes Entwicklungsmuster wahrscheinlich machen und Anpassungen an veränderte außenwirtschaftliche Bedingungen erschweren oder gar verunmöglichen.

Pauschal ist zunächst einmal zu vermuten, daß die nötigen, aber unterlassenen Anpassungsschritte in den betroffenen Ländern mindestens so unangenehme (kurz-bis mittelfristige) wirtschaftliche und politische Folgen hätten, als die steigenden Leistungsbilanzdefizite und Schuldenlasten. Anpassung ist ja ein Euphemismus für Konsumeinschränkungen, die aus der Uberwälzung der höheren internationalen Preise auf den Verbraucher resultieren. Dies bedeutet bei der geringen Flexibilität der Preise nach unten zunächst einmal geringere Produktion und damit Beschäftigung. Zur Rückgewinnung des externen Gleichgewichts und zur Wiederbelebung der Wirtschaft ist eine stärkere Verlagerung der Produktion für den Inlandsmarkt auf die Herstellung von Exportgütern vonnöten, was in der Regel Paritätsänderungen und Neuinvestitionen verlangt. Diese naturgemäß stark einkommensumverteilenden Maßnahmen finden in einer Umgebung statt, in der die verschiedenen gesellschaftlichen Gruppen ihre relative Einkommensposition zu erhalten trachten. Die politischen Anpassungsschwierigkeiten verschärfen sich noch

35 Kritische Literatur zur importsubstituierenden Industrialisierung würde Seiten füllen; eine neuere, pointierte Stellungnahme findet sich im World Development Report 1981.

36 Vgl. Anm. 1, S. 49 ff.

37 Siehe Tony Killick, Anm. 31. 
durch das zeitliche und gruppenmäßige Auseinanderfallen von Kosten und Gewinnen. Denn die mit dem Umschalten auf eine stärker exportorientierte Entwicklung verbundenen Preiserhöhungen und Einkommensverluste werden "in den politisch wichtigen städtischen Gebieten ${ }^{38}$ ja sofort sichtbar, während sich die Nutzen daraus nur mittelfristig und kaum für jene Unternehmen und Arbeitskräfte ergeben werden, die in bisher importsubstituierenden Branchen engagiert waren. Die von der möglichen Ausdehnung des Exports profitierenden Gruppen können von ihrem künftigen Glück allenfalls eine vage Ahnung haben und fallen daher als aktiv unterstützende Interessengruppe eines wirtschaftlichen Kurswechsels fort. ${ }^{39}$ In der einschlägigen Literatur werden Widerstände gegen die Liberalisierung des Handelsregimes gerne bei den organisierten Arbeitnehmern und den städtischen Massen in Entwicklungsländern geortet. Ein interessantes Modell der Weltbank zeigt aber, daß von den generell möglichen Anpassungspolitiken Währungsabwertungen relativ verteilungsneutral sind, die Importkontingentierung und ihre Beibehaltung aber zu einer deutlichen Begünstigung der Unternehmer (Renteneinkommen durch Lizenzen) führt. Bei einigermaßen realistischen Annahmen über die politische Machtverteilung drängt sich die Schlußfolgerung auf: "It would thus appear that devaluation is not a politically easy adjustment policy to implement " ${ }^{40}$ Erschwert und verlängert wird der Anpassungsprozeß noch in Zeiten geringer Ausdehnung des Welthandels und vor allem auch dann, wenn die mit der Handelsliberalisierung an sich verbundenen höheren Importe (etwa durch vergünstigte Entwicklungskredite) gar nicht finanziert werden können und der Ausgleich der Handelsbilanz allein über den Umweg der weiteren Nachfragedämpfung realisiert werden kann.

\section{Ländergruppen mit besonderen Anpassungsproblemen}

Länder die aus politischen und/oder wirtschaftlichen Gründen besondere Anpassungsprobleme an schwieriger werdende externe Bedingungen haben, wurden bisher nur etwas grobschlächtig als "binnenmarktorientiert" qualifiziert. Es handelt sich dabei um folgende, sich teils überschneidende Ländergruppen:

\section{a) Ärmere Entwicklungsländer}

Ihre Zahlungsbilanzdefizite sind heute bezogen. auf ihre Gütereinfuhren besonders hoch, obwohl diese (real) 1979 nur 2-3\% über denen von 1973 lagen und 1980 gar fielen. Weitere Importkompression würde ungebrochen auf die Produktion durchschlagen. Gleich-

$38 \mathrm{Vgl}$. Weltbank, Weltentwicklungsbericht 1980, S. 5 und ähnlich in den Weltentwicklungsberichten 1979 und 1981.

39 Siehe dazu Anne O. Krueger, Loans to Assist the Transition to Qutward-looking Policies, in: The World Economy, vol. 4, no. 3 (Sept. 1981).

40 Jaime de Melo, Trade Adjustment Policies and Income Distribution in Three Archetype Developing Economies, World Bank Staff Working Paper no. 442, Washington D.C., Dec. 1980. 
zeitig haben diese Länder ein für ihren Entwicklungsstand beängstigendes Schuldenniveau erreicht und haben ihre Währungsreserven nahezu verbraucht (1981). Auch unter der Annahme wirksamer Anpassungsschritte und erheblicher finanzieller Außenbeiträge kann diese Gruppe bis 1985 kaum mit realem Wirtschaftswachstum rechnen. ${ }^{41}$ Diese schwierige Lage ist teils durch die zur Zeit mäßige Nachfrage der Industrieländer und OPEC-Staaten nach ihren Produkten (meist agrarische Rohstoffe), mehr aber noch durch ihre bisherige Exportangebotsschwäche und die daraus folgenden Verluste an Marktanteilen und allgemein durch die nicht oder nur schwach steigende landwirtschaftliche Produktion - bei entsprechend höheren Nahrungsmittelimporten - bedingt. Die Ursachen hierfür liegen wiederum recht eindeutig in der übermäßigen Besteuerung der export crops - noch verstärkt durch überbewertete Währungen - in den zu geringen Getreide-Ankaufspreisen und der Ineffizienz der staatlichen Vermarktungsagenturen. ${ }^{42}$ Die künftige Anpassungsfähigkeit dieser Länder wird von der prioritären Ausrichtung der Entwicklungsstrategie auf die Landwirtschaft abhängen, was keineswegs industrielle Abstinenz bedeutet. Es zeigt sich nämlich, daß in jenen Ländern (Elfenbeinküste, Kenia, Malawi), wo ausreichende Produzentenpreise geboten wurden und vor allem die kleinen Grundbesitzer gefördert wurden, nicht nur die Nahrungsmittelproduktion, sondern auch die Industrialisierung am schnellsten voranschritt. ${ }^{43}$

Krisenverschärfend wirkte bei den ärmeren NOPECs die Stagnation der öffentlichen Entwicklungshilfe in der zweiten Hälfte der 70er Jahre hinzu. Trotz aller Beteuerungen der Geber, ihre Hilfe zunehmend am Kriterium der Hilfebedürftigkeit auszurichten, erhalten die ärmeren Länder nach wie vor deutlich geringere Mittel pro Kopf (etwa die Hälfte) als die "middle income countries". ${ }^{44}$

\section{b) Rohstof fexportierende Entwicklungsländer}

Der empirische Ländervergleich zur Bewältigung der externen Schocks nach 1974 zeigt, daß diese im Falle der rohstoffexportierenden Länder nur minimal durch strukturelle Anpassung, im wesentlichen aber durch die Aufnahme von Auslandskrediten erfolgte..$^{45}$ Dies ist auch nicht weiter verwunderlich, bedingt doch die Spezialisierung auf die Produktion und den Export mineralischer oder agrarischer Rohstoffe samt der begleitend notwendigen Infrastruktur eine hochgradig inflexible Produktionsstruktur und Verletzbarkeit gegenüber internationalen Nachfragetrends. Mit der oft beschriebenen zyklischen Entwicklung der Exporteinnahmen geht das Problem der mangelnden Neutralisierung von "windfall gains" in Boomzeiten einher bzw. der mangelnden Fähigkeit, die in

41 Vgl. Anm. 1; World Bank, World Development Report 1981, S. 54 ff.; IMF, Annual Report 1981, Washington D.C., 1981, S. $38 \mathrm{f}$.

42 Zur Zurechnung: World Development Report 1981, S. 66; World Bank, Accelerated Development in Sub-Saharan Africa - An Agenda for Action, Washington D.C., 1981.

43 Vgl. Weltbank, Weltentwicklungsbericht 1978, Washington D.C., 1978, S. 55 ff.

44 World Development Report, Anm. 42, S. 56.

45 Ebenda, Tabelle S. 66. 
besseren Zeiten begonnenen massiven Investitions- und Sozialprogramme auf ein in Baissezeiten finanzierbares Maß zu kürzen. Die Exporteure mineralischer Rohstoffe zeichnen sich dadurch aus, daß die Verbindung von Rohstoffsektor mit dem Rest der Wirtschaft wegen der stark unterschiedlichen Kapital- und Technologieintensität im wesentlichen nur über den Staatsapparat - via Steuereinzug und Investition in der Restwirtschaft - zuwege kommt; entscheidend ist bei dieser Scharnierfunktion des Staates, daß die Steuerabschöpfungen optimiert und in sorgfältig geplante Investitionsprogramme umgesetzt werden, die den sich ändernden Weltmarktbedingungen Rechnung tragen, daß also die wirtschaftspolitische Performanz der öffentlichen Institutionen der Entwicklung zuträglich ist.

Der Vergleich dieser Ländergruppe mit anderen zeigt, daß sie trotz günstigerer Ausgangsbedingungen (höheres Steueraufkommen, leichterer Zugang zu Devisen und ausländischen Krediten) eine z. T. deutlich schlechtere Performanz aufweisen. Auffällig ist bei der Gegenüberstellung

- die schlechtere Einkommensverteilung in den erzexportierenden Ländern;

- die geringere marginale Spar- und die höhere Konsumquote;

- die vor allem zu Zeiten von Rohstoffbaissen hohen Inflationsraten;

- das geringere Wachstum der Landwirtschaft;

- die praktisch nicht-existente Exportdiversifikation (1960-76) bei weit vorangetriebener, wenig effizienter und kapitalintensiver Importsubstitution;

- die höhere Außenverschuldung. ${ }^{46}$

Erzexportierende Länder können es sich wegen der leichteren Verfügbarkeit von Devisen und Auslandskrediten offenbar leisten, einen ineffizienten Industrialisierungsweg ohne Korrekturen länger durchzuhalten. Die zwangläufig größere wirtschaftliche Bedeutung des Staates in diesen Ländern trägt die Gefahr entsprechender Selbstprivilegierung der Staatsklassen in sich, die im Extremfall nur im verfügbaren Mehrprodukt ihre Grenze findet. ${ }^{47}$ Gleichzeitig sorgen die vom Rohstoffsektor alimentierten und begünstigten "vested interests" - zumal sie sich beim Enklavencharakter der Rohstoffwirtschaft und dem damit zusammenhängenden Staatsapparat (samt der öffentlichen Betriebe) leicht organisieren und mobilisieren lassen - dafür, daß eventuelle Strukturreformen mit erheblichen politischen/sozialen Friktionen verbunden sind.

\section{c) "Sozialistische" Entwicklungsländer}

Empirisch fundierte Ländervergleiche der letzten Jahre zeigen relativ deutlich, daß sich sozialistisch orientierte Entwicklungsländer - wobei "Sozialismus" in den Analysen alles von populistischen Regimen bis zu Zentralverwaltungswirtschaften einschließt - sich ge-

$46 \mathrm{Vgl}$. Gobind Nankani, Development Problems of Mineral-Exporting Countries, World Bank Staff Working Paper no. 354, Washington D.C., August 1979.

47 Siehe dazu Hartmut Elsenhans, Abhängiger Kapitalismus oder bürokratische Entwicklungsgesellschaft Versuch über den Staat in der Dritten Welt, Frankfurt/New York, 1981, Kap. V. 
genüber den stärker marktwirtschaftlich orientierten Ländern durch geringere Wachstumsraten (v. a. der verarbeitenden Industrie) auszeichnen. Damit zusammenhängend haben sich die Ausfuhren dieser Staaten in schwächerem Maße ausgeweitet und auch diversifiziert. Die geringe Exportdynamik führte bei den sozialistischen Ländern - bei hohem Importanstieg - zu einem rascheren Anstieg der Schuldendienstquote während der 70er Jahre als bei den marktwirtschaftlich orientierten Entwicklungsländern. ${ }^{48}$ Die geringere wirtschaftliche Dynamik wurde schließlich auch nicht - zumindest nicht wesentlich - durch signifikant bessere soziale Fortschritte kompensiert. ${ }^{49}$ Verantwortlich für die mäßige Kapitalproduktivität waren neben der genannten Importsubstitutionsstrategie die Niedrigzins- und Mindestlohnpolitik, v. a. aber auch die Úberfrachtung der zahlreichen Staatsbetriebe mit betriebsfremden Aufgaben und die direkten staatlichen Wirtschaftskontrollen. Staatsbetriebe in Entwicklungsländern operieren gemeinhin in gegenüber ausländischer Konkurrenz wohlgeschützten Binnenmärkten und erhalten prioritär Devisen- und Kreditzuteilungen sowie Steuervergünstigungen. Dennoch stellten sie meist nur Belastungen für die knappen Haushalte dar. Dies hat einmal damit zu tun, daß mit ihrer Etablierung nicht nur betriebswirtschaftliche, sondern auch sozial- und entwicklungspolitische Ziele verfolgt werden. Sie haben zudem wenig Autonomie bei der Festsetzung von Löhnen, Preisen und Investitionen und werden häufig als Arbeitsmarktpuffer mißbraucht. Die natürlicherweise sich einstellenden Verluste werden aus der Staatskasse beglichen, womit auch Anreize zur künftigen Leistungssteigerung untergraben werden. ${ }^{50}$

Was die Wirtschaftspolitik im weiteren Sinne anbetrifft, so beobachtet man bei sozialistischen/linkspopulistischen Regimen (vor allem in der Ubergangszeit nach einem eventuellen Regimewechsel) einen scharfen Fall der Arbeitsproduktivität, der zudem meist von gesetzlichen Erhöhungen der Mindestlöhne begleitet wird. Die an sich zu erwartenden Reaktionen des privaten Sektors (Preiserhöhungen, Entlassungen) werden durch staatliche Kontrollen unterbunden. Exportschwäche, Konkurse bzw. weitere Ưbernahmen durch den Staat, Kapitalflucht und Inflation durch Erhöhung der Konsumquote bei zurückgehendem Angebot, Zahlungsbilanzpassivierung und steigende Verschuldung sind dann nur noch die logische Folge. ${ }^{51}$

Die Schlußfolgerungen, die sich aus dem Bisherigen ergeben sind nun keineswegs, daß ein sozialistisch orientierter Entwicklungsweg von vornherein zum Scheitern verurteilt wäre, sondern nur, daß er denselben oder - wegen der zu erwartenden Kapitalflucht und "Zurückhaltung" der Kreditgeber - gar noch schärferen internen und externen Restriktionen unterliegt, wie ein stärker marktwirtschaftlich orientierter Weg. Lebensfähig wird ein sozialistischer Entwicklungsweg vermutlich dann, wenn die Zahl der verstaatlichten

48 Axel Halbach et al., Wirtschaftsordnung, sozio-ökonomische Entwicklung und weltwirtschaftliche Integration in den Entwicklungsländern, Studienreihe des BMWi, Bonn, März 1982.

49 Ebenda und David Morawetz, Economic Lessons from Some Small Socialist Developing Countries, in: World Development, vol. 8 (1980), S. 337-369.

50 Dazu: World Development Report 1979, S. 63 ff.

51 Siehe Morawetz, Anm. 49. 
Betriebe die administrative Kapazität des Landes nicht überschreitet, wenn die finanzielle und operative Autonomie der Staatsbetriebe gewährleistet wird und Sozialisierungen schnell und unter klarer Indentifikation der zu verstaatlichenden Betriebe bei expliziter Bestandsgarantie für die verbleibenden Privatunternehmen erfolgen. Staatliche Steuerung vertraut besser auf finanzielle Anreize (Steuern, Zölle) als auf direkte Kontrollen und generell ist eine eher konservative Außenwirtschafts- und Finanzpolitik die für ein stetiges Exportwachstum sorgt, allzu große Haushaltsdefizite, ein übermäßiges Geldmengenwachstum und negative reale Zinssätze vermeidet, empfehlenswert. Zuletzt ist auch deutlich, daß der mit dem Ubergang zu einer prononciert sozialistischen Wirtschaftspolitik verbundene Fall der Arbeitsproduktivität keine Zeit für umfangreiche Lohnerhöhungen ist; ein allerdings politisch nur schwer zu vermittelnder Tatbestand.

\section{d) Länder mit schlechtem "politischen Krisenmanagement "}

Ein großer Teil jener Literatur, die sich mit Fragen außenwirtschaftlicher Anpassung beschäftigt, behandelt die notwendige politische Einleitung und Umsetzung von entsprechenden Programmen, die damit verbundene Mobilisierung breiter gesellschaftlicher Unterstützung und die Geschicklichkeit bei der Allokation von Anpassungslasten eventueller Kurskorrekturen als minder wichtige Probleme. ${ }^{52}$ Dies ist aber keinesfalls gerechtfertigt. Im Gegenteil kann man zeigen, daß die Stärkung stabilitätsfördernder politischer Institutionen und Verhaltensweisen und vor allem die politische Glaubwürdigkeit von Kurskorrekturen meist weitaus bedeutsamer sind als das Erreichen kurzfristiger und willkürlicher ökonomischer "performance targets". Wie noch auszuführen sein wird, ist das Entscheidende bei Anpassungsprogrammen, daß sie politisch so lange durchgehalten werden, bis sich erste sichtbare wirtschaftliche Erfolge einstellen. Dabei kommt es in erster Linie auf die Glaubwürdigkeit der Regierung an. Ein Umschwenken auf eine stärker außenorientierte Wirtschaftspolitik bringt zunächst einmal Produktionskürzungen bei den importsubstituierenden Industrien. Sind nun die Unternehmer von der Durchhaltefähigkeit des neuen Kurses nicht überzeugt, werden sie sich bei Investitionen in der Exportproduktion zu- und stattdessen mit Importgütern, Immobilien o. ä. spekulieren. Damit verlängert und intensiviert (Arbeitslosigkeit!) sich aber der Anpassungsprozeß und folgerichtig die gegen ihn gerichteten Widerstände. Bei einem eventuell vorzeitigen Abbruch des neuen Kurses sinkt dann auch die Glaubwürdigkeit jedes künftigen Stabilisierungsexperimentes. ${ }^{53}$

Wie vor allem lateinamerikanische Fälle demonstrieren, kann daraus leicht ein Teufelskreis ständig repressiverer nichtsdestoweniger aber nur mäßig erfolgreicher Stabilisie-

52 Vgl. Alejandro Foxley und Laurence Whitehead, Economic Stabilization in Latin America: Political Dimensions - Editor's Introduction, in: World Development, vol. 8 (1980), S. 823-832.

53 Ebenda und William R. Cline und Sidney Weintraub, Introduction and Overview, in: dies., Economic Stabilization in Developing Countries, a.a.O. Im selben Band: Anne O. Krueger, Interactions Between Inflation and Trade Regime Objectives in Stabilization Programs. 
rungsprogramme entstehen. Vorbedingungen eines erfolgreichen politischen Krisenmanagements scheinen vor allem effektive Redistributionsinstanzen zu sein, die in den Augen der betroffenen Gruppen zur akzeptablen intertemporalen Allokation der Anpassungslasten in der Lage sind, sowie eine autonome Problemverarbeitungskapazität, die es den Regierungen erlaubt, ein eigenständiges, nicht nur von außen (IMF) aufgenötigtes Sanierungskonzept zu repräsentieren. Etwas spekulativ, scheinen auch Entwicklungsgesellschaften mit relativ geringer Einkommenskonzentration eher in der Lage, sich an verschlechterte externe Bedingungen anzupassen.

Das gerne gehörte Vorurteil, nur Militärdiktaturen seien zur Umsetzung durchgreifender Korrekturen in der Lage, ist schlicht falsch. Sie verfügen meist nur über eine deutlich höhere Repressionskapazität, die freilich im Zeitablauf (Paradebeispiel: Argentinien) sinkenden Grenznutzen aufweist. Der Zusammenhang ist vielmehr umgekehrt: Länder, die die instabilste wirtschaftliche Entwicklung aufweisen, produzieren auch die schlimmsten politischen Regime. ${ }^{54}$

\section{Lösungsmöglichkeiten}

Wenn also externe Gründe für die Verschlechterung der außenwirtschaftlichen Lage der NOPECs im Gefolge der beiden Olkrisen keineswegs unbedeutend waren, so kommt der effektiven internen wirtschaftspolitischen Verarbeitung doch offenbar ein größeres Gewicht zu. Das bedeutet nun keineswegs, die besonders gefährdeten Länder sollten allein durch Selbsthilfe - mittels eines engeren Gürtels - die Krise meistern:

a) Die oben angeführten Untersuchungen zeigen teilweise, daß Krisenanpassungsstrategien, die allein auf Nachfragebeschränkungen bzw. Wachstumsverzicht hinausliefen, relativ ineffizient waren..$^{55}$ Gelungene Anpassung zeichnete sich in der weit überwiegenden Mehrzahl der Fälle durch eine Kombination kurzfristiger nachfragedämpfender Maßnahmen und strukturverändernder Wirtschaftspolitiken (Exportexpansion, Beseitigung sektoraler Engpässe, Sparanreize etc.) aus. Die mangelnde Wirksamkeit von Wachstumsdrosselung allein ist auch unmittelbar einleuchtend, da sie weder Rückzahlungsfähigkeit noch Kreditwürdigkeit stärkt.

b) Die vorliegenden Ländervergleiche zeigen auch, daß v. a. die Schwellenländer bzw. die industriell stärker diversifizierten NOPECs zu einer wachstumserhaltenden, strukturverändernden Wirtschaftspolitik in der Lage waren. Schon per definitionem erlaubt eine fortgeschrittenere Wirtschaftsstruktur eine leichtere Umlenkung der Produktionsfaktoren auf den Export, ganz abgesehen davon, daß sie auch besseren Zugang zu externem Kapital garantiert. ${ }^{56}$

54 Siehe Foxley/Whitehead, Anm. 52 und Alejandro Foxley, Stabilization Policies and their Effects on Empolyment and Income Distribution: A Latin American Perspective, in: Cline/Weintraub, Anm. 53.

55 Dazu vor allem Jaspersen, Anm. 29, S. 15 ff.

56 Ebenda und Veronika Büttner, a.a.O., S. 41. 
c) Die Problemländer haben ihre "Anpassungsressourcen" (interne Ersparnisse, Importkompression etc.) meist bereits vollständig verbraucht, wenn es zu einer Zahlungsbilanzkrise kommt und zwar ohne ein sonderliches Wirtschaftswachstum erreicht zu haben, daß die Anpassungslasten sozial erträglich und ihre Verteilung politisch konsensfähig machen könnte.

d) Die Schwierigkeiten und sozialen Kosten des Umschaltens auf eine offenere Wirtschaftspolitik nehmen mit Dauer und Intensität des bisher verfolgten Kurses zu, daß ja parallel die hiervon profitierenden Unternehmen, Arbeitskräfte und die sie betreuenden Bürokratien an Macht und Einfluß gewinnen. Zudem verlängert sich der Anpassungszeitraum parallel zur Intensität der praktizierten Importsubstitution. Die Schwierigkeit ist nun ein glaubwürdiges Programm vorzulegen (s. o.) und während seiner Laufzeit dem Druck jener Gruppen standzuhalten, die Verluste erleiden. Daraus ergibt sich zweierlei:

Einmal dürfen die mit und ohne Hilfe des IMF durchgeführten Anpassungsprogramme in jenen Problemländern nicht so hart sein, daß sie aus politischen Gründen abgebrochen werden müssen, bevor sie Erfolge zeitigen können um die Chancen künftiger Programme, damit aber auch der dauerhaften wirtschaftlichen und politischen Stabilität in den betroffenen Ländern zu wahren.

Zum zweiten ist ein Ubergang zu einem liberalen Außenhandelsregime dann schwierig, wenn die zwangsläufig steigenden Importe nur über einen scharfen wirtschaftlichen Restriktionskurs begrenzt werden können. ${ }^{57}$ Beides spricht dafür, die Anpassung mit ausreichenden und gezielt eingesetzten Mitteln der Entwicklungshilfe (oder des IMF) zu erleichtern und damit Sorge zu tragen, daß sie auch ohne die Zwischenschaltung repressiver Militärregime fortgesetzt werden kann.

e) Simulationsmodelle der Weltbank zeigen, daß risikoreiche Verschuldungslagen sich in strukturelle gefährdeten Entwicklungsländern sehr rasch aufbauen. Erfolgen wirtschaftspolitische Korrekturen erst kurz (d. h. immerhin drei Jahre) vor Erreichen der kritischen Verschuldungsgrenze, so ist das Risiko einer Verschuldungskrise auch durch extreme Schritte nicht mehr signifikant zu verringern. ${ }^{58}$ Dies spricht zusammen mit der bereits angesprochenen Stärkung jener Kräfte im Zeitablauf, die durch Änderungen zu verlieren haben, und der mit dem Hinausschieben verbundenen Intensivierung der Korrekturen, für ihr rechtzeitiges Einleiten. Da Politiker sich systembedingt auf die kurzfristig anfallenden Lasten konzentrieren, wäre zu überlegen, wie das internationale Instrumentarium so ausgestattet werden kann, daß frühzeitige Anpassung prämiert wird. Zur Zeit ist leider das Gegenteil der Fall:

- Die internationalen Bankkredite an die Problemländer zeichnen sich durch eine ausgesprochene Prozyklizität aus: zu Zeiten großer Liquidität der Euromärkte werden diese Länder, da auch noch kreditwürdig, mit Krediten geradezu überschüttet. Die Folge ist dann oft die Stimulierung eines Konsum- und Investitionsniveaus bei den Kreditneh-

$57 \mathrm{Vgl}$. Anne O. Krueger, Anm. 39.

58 Siehe dazu die Studie von Gershon Feder, Economic Growth, Foreign Loans and Debt Servicing Capacity of Developing Countries, World Bank Staff Working Paper no. 274, Washington D.C., Feb. 1978. 
mern, das später nur schwer reduziert werden kann. Wenn sich die Zahlungsbilanzsituation der Länder verschlechtert, setzt ein abrupter Fall der Bruttoneukredite ein, der dazu führt, daß diese Staaten in dem Augenblick, wo sie neuer Mittel am ehesten bedürftig sind, oftmals auch einen Kapitalrückfluß finanzieren müssen. Der Eindruck, daß in einigen Fällen zu viel, zu lange und zu sorglos geliehen wurde, läßt sich nicht von der Hand weisen. Zum Teil lag dies daran, daß die Banken hofften, die öffentlichen Kreditgeber würden schon rechtzeitige Schritte unternehmen (Umschuldungen im Rahmen des Pariser Klub) um ihre Risiken vermindern zu helfen oder aber strategisch wichtige Länder (Türkei) über Wasser zu halten. Entsprechend ärgerlich reagierten sie denn zuweilen auf die sonst immer geforderten frühen Warnungen des IMF. ${ }^{59}$

Die lange Verfügbarkeit von privaten Krediten machte es den betroffenen Ländern auch möglich, an sich nötige Anpassungsschritte hinauszuschieben und den Gang zum IMF zu meiden, damit aber umso sicherer (s. o.) die Krise herbeizuführen.

- Der frühe Gang zum IMF unterbleibt möglicherweise nicht nur wegen der Schwere der erwarteten Auflagen, sondern auch der nur vergleichsweise geringen Mittel, die dafür eingehandelt werden. Gemessen am aggregierten Leistungsbilanzdefizit der Entwicklungsländer sind die IMF-Ressourcen trotz wiederholter Aufstockung ständig (v. a. im auflagenfreien Bereich) gesunken. Auch kann vielfach beobachtet werden, daß die Hoffnung auf die einem IMF-Stabilisierungsprogramm nachfolgenden Bankenkredite (als Folge der wiederhergestellten Kreditwürdigkeit) sich oft in geringerem Maße bewahrheitet haben, wie die jeweiligen Entwicklungsländer dies wohl erhofft haben mögen.

Daraus ergibt sich, daß der IMF bei der Defizitfinanzierung der Entwicklungsländer, speziell der Problemfälle, in Zukunft sicher eine bedeutendere Rolle übernehmen muß. ${ }^{60}$ Mit der Revision der Quoten, der Ziehungsmöglichkeiten und der Vergaberichtlinien 1979 - die sich in einer substantiellen Erhöhung der Ausleihungen 1981 niederschlugen wurde hierzu bereits ein großer Schritt getan. Parallel zu der vielfach geforderten prominenteren Rolle von Währungsfonds und Weltbank bei der Defizifinanzierung von Entwicklungsländern ist auch viel von bürgerschaftsähnlichen Absicherungen der Bankkredite an die Kreditnehmer durch den IMF (oder bilaterale Organe) oder von einem verstärkten Gebrauch von Kofinanzierungen (Banken plus Weltbank) zur Risikominderung die Rede. Von diesen Vorschlägen ist - ohne hier näher auf Details eingehen zu wollen insgesamt nicht viel zu halten. Der weitere Ausbau des Garantieinstrumentariums - auf nationaler Ebene besteht eine Fülle von Fazilitäten - würde neben einer weiteren Sozialisierung der Verluste nur die nötige Sorgfalt der Banken bei der Kreditvergabe mindern. Seltsamerweise verlangen stets jene neue Garantien, die behaupten, die Entwicklungsländer seien bereits total überschuldet. ${ }^{61}$ Die vernünftigere Alternative wäre wohl eine adäquate Reservebildung der Banken zur Abdeckung der Ausfallrisiken. ${ }^{62}$

59 Vgl. Cline/Weintraub, Anm. 53, S. 40; Anm. 1, S. 39; Group of Thirty, Anm. 17, S. 9.

60 Statt vieler: Rolf M. Jeker, Conditionality and Stand-by Credits of the International Monetary Fund and Less Developed Countries, in: Außenwirtschaft, Jg. 35, Heft I (März 1980).

61 So in Group of Thirty, Anm. 17, S: 28.

62 Wie sie der Economist (Anm. 23) vorschlägt. 
- Zuletzt wird der Spielraum der hochverschuldeten Länder aber auch durch Inflexibilitäten der öffentlichen Entwicklungshilfe beschränkt: der Anteil der liefergebundenen Hilfe bei den bilateralen Zuwendungen ist nach wie vor erheblich (ca. $60 \%$ ) und der Anteil der projektgebundenen Hilfe ist deutlich im Steigen begriffen (1976: $55 \%$; 1979: $72 \%) \cdot{ }^{63}$ Projekthilfe bindet in einem erheblichen Maße Ressourcen des Empfängers und zwingt ihn bei auftretenden Zahlungsbilanzproblemen (wegen der mangelnden Flexibilität des Mitteleinsatzes) auf kurzfristige und teure Privatkredite zurückzugreifen. ${ }^{64}$ Die außenwirtschaftlichen Probleme einer ganzen Reihe von Ländern (Prototyp: Tansania) sind von der Vielzahl der in Angriff genommenen Entwicklungshilfeprojekte zumindest mitverursacht worden.

f) Die uneingeschränkte Empfehlung stärker exportorientierter Strategien, die Balassa und seine Mitarbeiter aus den empirischen Ländervergleichen für die Jahre 1974-78 gezogen haben, kann von zwei Ansatzpunkten her in Frage gestellt werden:

- Strukturalistische Theoretiker bezweifeln angesichts der rigiden Produktionsstrukturen in Entwicklungsländern, der geringen Einkommens- und Preiselastizitäten der Nachfrage nach den Exportgütern dieser Länder in den OECD-Staaten und der mangelnden weiteren Komprimierbarkeit der hauptsächlich aus Zwischen- und Investitionsgütern bestehenden Importe am Erfolg der üblichen exportsteigernden Instrumente (Nachfragedrosselung, Abwertung). Dies wird durch die Erfahrung der Stabilisierungsprogramme in den 70er Jahren (z. B. Uruguay, Chile, Portugal), die alle von einer beeindruckenden Steigerung der nichttraditionellen Exporte begleitet waren, nicht bestätigt: "Evidence indicates a clear victory for export optimists", resümiert Diaz-Alejandro, selbst früher Anhänger der strukturalistischen Schule. ${ }^{65}$

- Augenscheinlich hängen die Aussichten weiterer Entwicklungsländer eine erfolgreiche exportorientierte Strategie zu verfolgen, von der Aufnahmefähigkeit der Weltmärkte, speziell den protektionistischen Tendenzen in den westlichen Industrieländern ab. Uber die zunehmende Abschottung dieser Märkte in den letzten Jahren mangelt es nicht an Klageliedern; dabei wird oft vergessen, daß die Fertigwarenausfuhren der Entwicklungsländer in dieser Zeit rasch zugenommen haben und sie ihren Marktanteil in den OECD-Staaten deutlich ausweiten konnten. Dennoch wird man feststellen müssen, daß bei den augenblicklichen Wachstumsaussichten des Welthandels, der Export für die "Spätankömmlinge" sicher nicht die gleiche Rolle spielen kann, wie für einige südostasiatische Länder.66

63 OECD, Development, Co-operation, 1980 Review, Paris, 1980, S. 105.

$64 \mathrm{Vgl}$. Helen B. Junz, How to Assure the Stability of the Financial System, in: The World Economy, vol. 4, no. 3 (Sept. 1981), S. 268.

65 Carlos F. Diaz-Alejandro, Southern Cone Stabilization Plans, in Cline/Weintraub, Anm. 53, S. 125.

66 Dazu: William R. Cline, Canthe East Asian Model of Development Be Generalized? in: World Development, vol. 10, no. 2 (Febr. 1982); Alfred Pfaller, What Can LDCs Expect From Export oriented Industrialization? in: Vierteljahresberichte Nr. 75, 1979; Helen Hughes und Jean Waelbroeck, Can Developing-country Exports Keep Growing in the 1980s? in: The World Economy, vol. 4, no. 2 (June 1981). 
Dies ist vor allem auch deswegen der Fall, weil das Lohnniveau bei den prospektiven Spätankömmlingen - vor allem den afrikanischen Ländern - nicht sonderlich niedrig liegt und vermutlich auch gar nicht so weit gedrückt werden kann um die sonstigen Standortnachteile (mangelnde Infrastruktur, zu wenig ausgebildete Fachkräfte etc.) auszugleichen.

- Der Úbergang zu einer exportorientierten Strategie bedarf einer entsprechenden gesellschaftlichen Basis. Es ist auffällig, daß vor allem sich entwickelnde Volkswirtschaften mit einer relativ gleichmäßigen Einkommensverteilung Exporterfolge aufweisen (v. a. die südostasiatische "Viererbande") und daß Regierungen in Ländern mit extremen sozialen Unterschieden (besonders in Lateinamerika) ihre Offnungspolitik in dem Augenblick zu lockern begannen, wie sich nur eine einigermaßen erträgliche Zahlungsbilanz einstellte. 
ties and are affected by their political swings and outside advice. However, they largely express the needs and hopes of their members. - Finally, the dilemma of those officals is discussed, who want to organize the peasantry for their economic and social promotion, but without any political activity. This patronizing approach is doomed to failure.

\section{Indeptedness, Indeptedness Crisis and Adaptability of Developing Countries}

\section{By Joachim Betz}

According to this essay there is no generalized debt problem of developing countries nor severe capital constraints by the international banks (due to portfolio constraints, lack of equity capital or mounting regulation) to service them on past volume standards. The extrenal debt of some developing countries will, however, become unmanageable, if the present global recession continues and if they are not undertaking serious and far-reaching adjustment efforts.

Developing countries have shown in the past - particularly after the firts oil crisis - fairly different adjustment capabilities to deteriorating external economic conditions. The countries with the poorest record are those mainly commodity exporting countries, who have pursued import substitution strategies beyond the first stage and who have relied heavily on indiscriminate state regulation of the economy. Even if external causes are of secondary importance for their worsening resource balance, it would nevertheless be dangerous to expect those countries to improve their situation by belt-tightening alone. This would neither be sufficient nor politically feasible. There is such an urgent necessity to assist the adjustment efforts of the hard core problem countries of the Third World, if continuing and cumulative debt crisis are to be avoided. 